\title{
Interactive comment on "PHIPS-HALO: the airborne particle habit imaging and polar scattering probe - Part 3: Single Particle Phase Discrimination and Particle Size Distribution based on Angular Scattering Function" by Fritz Waitz et al.
}

\section{Greg McFarquhar (Referee)}

mcfarq@ou.edu

Received and published: 25 October 2020

Review of "PHIPS-HALO: the airborne particle habit imaging and polar scattering probe- Part 3: Single particle phase discrimination and particle size distribution based on angular scattering function" by Waitz et al. 
This study uses data collected by the PHIPS-Halo during two field campaigns (ACLOUD and SOCRATES) to develop a method to determine the phase of individual cloud hydrometeors from the light scattering measurements made by the probe. The algorithm takes advantage of differences in features between the angular scattering of spherical and aspherical particles to show that it can be determined with a $98 \%$ accuracy whether the particles are liquid or ice (i.e., spherical or aspherical). They also present a method for deriving the particle size distributions from the measured data. Information about particle phases is desperately needed because mixed-phase clouds are still not well understood. Further, the full potential of the PHIPS-HALO probe has yet to be realized because both particle size distributions and particle phase distributions have not been routinely been made available in a short time period after the conduct of field campaigns. As the material in this paper works to overcome both of these shortcomings, it should be published as soon as possible and represents a good contribution to the refereed literature. The paper is well written and technically sound so does not require a lot of revisions in that respect. Nevertheless, I think there are a couple of aspects that should be better explained in the paper so that the limitations, as well as the strengths, of the PHIPS-HALO for providing size and habit information are well outlined.

My major critique of the paper is that I think more information about the statistical representativeness of the data that are available from the PHIPS HALO probe should be included. The sample volume and/or sample area of the PHIPS-HALO probe should be explicitly stated. How does that compare against the sample volume/area from the commonly used. How does that affect the averaging time over which representative particle size distributions and particle phase distributions are available? For example, McFarquhar et al. (2007) calculate the required time that particle size distributions would need to be averaged over in order to obtain statistically significant particle size distributions that they defined to mean 100 particles in each size bin (so that there was a $10 \%$ uncertainty assuming the statistical uncertainty was proportional to the square root of the number of counts in each size bin). Figure 9 defines the sensitive area of

Interactive comment 
the PHIPS-HALO as ranging from $0.002 \mathrm{~cm}^{\wedge} 2$ to about $0.01 \mathrm{~cm}^{\wedge} 2$ depending on the size of the particle. Assuming a roughly $150 \mathrm{~m} / 2$ air speed, this would give sample volumes ranging from 30 to $150 \mathrm{~cm}^{\wedge} 3$ per second, or about 0.15 liter/second. This would seem to be quite a bit less than that of the 2DS/2DC class of probes. This does not negate the benefits of the PHIPS-HALO probe, but rather would seem to suggest that the more detailed phase/shape information available from the PHIPS-HALO probe has synergy with the more frequent data available from the optical array type probes that are better suited for deriving the fine resolution structure of clouds. This point, including explicitly comparing the sampled areas/volumes and numbers of particles between probes should be explicitly shown in the paper, and also mentioned in the abstract.

\section{Detailed Comments}

Page 1, line 6: evaluated would be a better word than validated. Page 2, line 13: "a large sampling statistics is required" reads awkwardly and should be rephrased. Page 3, line 15: the plural of aircraft is aircraft. Page 4, line 7: Suggest adding Um et al. 2011 ACP to the list of references as they considered scattering functions of several models of quasi-spherical ice crystals Page 4, Figure 2: Can you state what are the maximum dimensions of the two ice crystals that are considered in the figure? Page 7, line 23: It should be noted and discussed why there are a lot of differences in the nature of the distributions between the observed and modeled particles in Figures 4 and 5. Does this suggest that there are some limitations in how well the theoretical models are representing the actual observed particles? Page 9, line 3 . This may be a stylistic thing, but when I see 41.000 I think there are 41 particles. I think the authors mean 41,000 . Unless this convention is demanded by ACP, I would use a comma rather than a period. Page 9, line 3: Related to my major point above, I think it would be very interesting to compare the number of particles that were measured by the 2DC/2DS for the same periods during these field projects. That would help clarify information about the statistical representativeness of the data. Page 11, Figure 6.

\section{AMTD}

Interactive

comment
Printer-friendly version

Discussion paper 
Was any effort made to go back and look at the particles that were misidentified to determine why they were misidentified? I agree that $98 \%$ is outstanding (and better than classifications that are based on other probe data), but it would still be interesting to know why the discrepancy for just these few particles. Was there any chance that the manual identification of these particles was incorrect? Page 13, Figure 8. Most probes that measure small particles have smaller sensitive areas for smaller particles than larger particles (e.g., see Figure 9 in this paper). But, as I understand it the vertical axis here is number of particles rather than some measure of concentration per bin or number distribution function. It would be informative to include another plot that shows the calculated number distribution function since that is a physically meaningful quantity, especially since the caption reads that this is a particle size distribution (PSD). Page 18, Figure 11. The caption should specifically state the averaging period for which each of the plotted points corresponds to. Page 22, Line 10. To make this study more accessible, it would be nice to have the codes used available on github or some other code repository.

Interactive comment on Atmos. Meas. Tech. Discuss., doi:10.5194/amt-2020-297, 2020. 